\title{
On Feasible Sets of Mixed Hypergraphs
}

\author{
Daniel Král'* \\ Department of Applied Mathematics and \\ Institute for Theoretical Computer Science ${ }^{\dagger}$ \\ Charles University, \\ Malostranské náměstí 25 \\ 11800 Praha 1, Czech Republic \\ kral@kam.ms.mff.cuni.cz
}

Submitted: Nov 20, 2003; Accepted: Feb 24, 2004; Published: Mar 4, 2004.

MR Subject classifications: Primary 05C15, Secondary 05C65, 05C85

Keywords: coloring of hypergraphs, mixed hypergraphs, algorithms for coloring

\begin{abstract}
A mixed hypergraph $H$ is a triple $(V, \mathcal{C}, \mathcal{D})$ where $V$ is the vertex set and $\mathcal{C}$ and $\mathcal{D}$ are families of subsets of $V$, called $\mathcal{C}$-edges and $\mathcal{D}$-edges. A vertex coloring of $H$ is proper if each $\mathcal{C}$-edge contains two vertices with the same color and each $\mathcal{D}$-edge contains two vertices with different colors. The spectrum of $H$ is a vector $\left(r_{1}, \ldots, r_{m}\right)$ such that there exist exactly $r_{i}$ different colorings using exactly $i$ colors, $r_{m} \geq 1$ and there is no coloring using more than $m$ colors. The feasible set of $H$ is the set of all $i$ 's such that $r_{i} \neq 0$.

We construct a mixed hypergraph with $O\left(\sum_{i} \log r_{i}\right)$ vertices whose spectrum is equal to $\left(r_{1}, \ldots, r_{m}\right)$ for each vector of non-negative integers with $r_{1}=0$. We further prove that for any fixed finite sets of positive integers $A_{1} \subset A_{2}\left(1 \notin A_{2}\right)$, it is NP-hard to decide whether the feasible set of a given mixed hypergraph is equal to $A_{2}$ even if it is promised that it is either $A_{1}$ or $A_{2}$. This fact has several interesting corollaries, e.g., that deciding whether a feasible set of a mixed hypergraph is gapfree is both NP-hard and coNP-hard.
\end{abstract}

\section{Introduction}

Graph coloring problems are intensively studied both from the theoretical point view and the algorithmic point of view. A hypergraph is a pair $(V, \mathcal{E})$ where $\mathcal{E}$ is a family of subsets

*The author acknowledges partial support by GAČR 201/1999/0242, GAUK 158/1999 and KONTAKT 338/99.

${ }^{\dagger}$ Institute for Theoretical Computer Science is supported by Ministry of Education of Czech Republic as project LN00A056. 
of $V$ of size at least 2. The elements of $V$ are called vertices and the elements of $\mathcal{E}$ are called edges. A mixed hypergraph $H$ is a triple $(V, \mathcal{C}, \mathcal{D})$ where $\mathcal{C}$ and $\mathcal{D}$ are families of subsets of $V$ of size at least 2. The elements of $\mathcal{C}$ are called $\mathcal{C}$-edges and the elements of $\mathcal{D}$ are called $\mathcal{D}$-edges. A proper $\ell$-coloring $c$ of $H$ is a mapping $c: V \rightarrow\{1, \ldots, \ell\}$ such that there are two vertices with $\mathcal{D}$ ifferent colors in each $\mathcal{D}$-edge and there are two vertices with a $\mathcal{C}$ ommon color in each $\mathcal{C}$-edge. A proper coloring $c$ is a strict $\ell$-coloring if it uses all $\ell$ colors. A mixed hypergraph is colorable if it has a proper coloring. Mixed hypergraphs were introduced in [23]. The concept of mixed hypergraphs can find its applications in different areas, e.g. list-coloring of graphs [14], graph homomorphisms [9], coloring block designs $[2,3,16,17,18]$, etc. The importance and interest of the concept is witnessed by a recent monograph on the subject by Voloshin [21]. As an example, we present here the following construction described in [14]: Let $G$ be a graph and let $L$ be a function which assigns each vertex a set of colors. A coloring $c$ of $G$ is a proper list-coloring with respect to the lists $L$ if $c(v) \in L(v)$ for each vertex $v$ of $G$ and $c(u) \neq c(v)$ for each edge $u v$ of $G$. Let $\mathcal{L}$ be the union of the lists of all the vertices of $G$. Consider a mixed hypergraph $H$ with the vertex set $V(G) \cup \mathcal{L}$ and the following edges: a $\mathcal{D}$-edge $\{u, v\}$ for each $u v \in E(G)$, a $\mathcal{D}$-edge $\{x, y\}$ for any $x, y \in \mathcal{L}(x \neq y)$ and a $\mathcal{C}$-edge $\{v\} \cup L(v)$ for each vertex of $G$. $H$ has a proper coloring iff $G$ has a proper list-coloring. Similar constructions have been found by the author [9] for graph homomorphisms, the channel assignment problem, $L(p, q)$-labelings of graphs and some other graph coloring problems.

The feasible set $\mathcal{F}(H)$ of a mixed hypergraph $H$ is the set of all $\ell$ 's such that there exists a strict $\ell$-coloring of $H$. The (lower) chromatic number $\chi(H)$ of $H$ is the minimum number contained in $\mathcal{F}(H)$ and the upper chromatic number of $\bar{\chi}(H)$ of $H$ is the maximum number. The feasible set of $H$ is gap-free (unbroken) if $\mathcal{F}(H)=[\chi(H), \bar{\chi}(H)]$ where $[a, b]$ is the set of all the integers between $a$ and $b$ (inclusively). If the feasible set of $H$ contains a gap, we say it is broken. The spectrum of a mixed hypergraph $H$ is the vector $\left(r_{1}, \ldots, r_{\bar{\chi}(H)}\right)$ where $r_{\ell}$ is the number of different strict $\ell$-colorings of $H$. Two colorings $c_{1}$ and $c_{2}$ are considered to be different if there is no permutation of colors changing one of them to the other, i.e., it is not true that $c_{1}(u)=c_{1}(v)$ iff $c_{2}(u)=c_{2}(v)$ for each two vertices $u$ and $v$. We remark that the spectrum is usually defined to be a vector $\left(r_{1}, \ldots, r_{n}\right)$ where $n$ is the number of vertices of the mixed hypergraph, but we prefer using the definition without trailing zeroes in the vector. If $\mathcal{F}$ is a set of positive integers, we say that a mixed hypergraph $H$ is a realization of $\mathcal{F}$ if $\mathcal{F}(H)=\mathcal{F}$. A mixed hypergraph $H$ is a one-realization of $\mathcal{F}$ if it is a realization of $\mathcal{F}$ and all the entries of the spectrum of $H$ are either 0 or 1 .

A necessary and sufficient condition on a set of positive integers to be the feasible set of a mixed hypergraph was proved in [6]:

Theorem 1 A set $\mathcal{F}$ of positive integers is a feasible set of a mixed hypergraph iff $1 \notin \mathcal{F}$ or $\mathcal{F}$ is an interval. If $1 \in \mathcal{F}$, then all the mixed hypergraphs with this feasible set contain only $\mathcal{C}$-edges.

In particular, there exists a mixed hypergraph such that its feasible set contains a gap. On the other hand, it was proved that feasible sets of mixed hypertrees [10], mixed 
strong hypercacti [13] and of mixed hypergraphs with maximum degree two [11, 12] are gap-free. Feasible sets of mixed hypergraphs with maximum degree three need not to be gap-free. The feasible sets of planar mixed hypergraphs, i.e., hypergraphs whose bipartite incidence graphs of their vertices and edges are planar [4, 15], are exactly intervals $\left[k_{1}, k_{2}\right], 1 \leq k_{1} \leq 4, k_{1} \leq k_{2}$ and sets $\{2\} \cup[4, k], k \geq 4$ as proved in [7].

Necessary or sufficient conditions for a vector to be a spectrum of a mixed hypergraph were not addressed in detail so far. However, Voloshin [22] conjectured a sufficient condition for a vector to be the spectrum of a mixed hypergraph (Conjecture 2 in [22]): If $n_{0}, \ldots, n_{t}$ is a sequence of positive integers such that $n_{i} \geq\left(n_{i-1}+n_{i+1}\right) / 2$ for $1 \leq i \leq t-1$ and $\max \left\{n_{\lfloor t / 2\rfloor}, n_{\lceil t / 2\rceil}\right\}=\max _{0 \leq i \leq t}\left\{n_{i}\right\}$, then there exists a mixed hypergraph $H$ such that $\chi(H)+t=\bar{\chi}(H)$ and $H$ allows exactly $n_{i}$ different strict $(\chi(H)+i)$-colorings $(0 \leq i \leq t)$. We prove this conjecture. In fact, Theorem 3 implies that the only hypothesis needed is that $n_{0}, \ldots, n_{t}$ is a sequence of non-negative integers. Let us remark at this point that Conjecture 1 from [22] on co-perfect mixed hypergraphs was disproved in [8].

We study several problems posed in [22] (Problem 10, 11, Conjecture 2) and in [6]. In particular, we are interested in the size of the smallest (one-)realization of a given feasible set. In [6], two constructions of a mixed hypergraph with a given feasible set $\mathcal{F}$ are presented, but both of them can have exponentially many vertices in terms of $\max \mathcal{F}$ and $|\mathcal{F}|$. The second construction from [6] does not even give one-realization of $\mathcal{F}$. We present an algorithmic construction (Theorem 2) which gives a small one-realization for a given feasible set $\mathcal{F}$. The number of vertices of this realization is at most $|\mathcal{F}|+2 \max \mathcal{F}-1$ and the number of edges is cubic in the number of vertices.

Theorem 2 from Section 2 can be restated as follows: Let $\left(r_{1}, \ldots, r_{m}\right)$ be a vector such that $r_{1}=0$ and $r_{i} \in\{0,1\}$ for $2 \leq i \leq m$. Then, there exists a mixed hypergraph $H$ such that the spectrum of $H$ is $\left(r_{1}, \ldots, r_{m}\right)$. Note that the condition $r_{1}=0$ is the condition $1 \notin \mathcal{F}$ mentioned earlier. We generalize this theorem in Section 3. We prove that for each vector $\left(r_{1}, \ldots, r_{m}\right)$ of non-negative integers such that $r_{1}=0$ there exists a mixed hypergraph such that its spectrum is equal to $\left(r_{1}, \ldots, r_{m}\right)$ (Theorem 3 ). The number of vertices of the mixed hypergraph from Theorem 3 is $2 m+2 \sum_{i=1, r_{i} \neq 0}^{m}\left(1+\left\lfloor\log _{2} r_{i}\right\rfloor\right)$ and the number of its edges is cubic in the number of its vertices. Theorem 3 provides an affirmative answer to Conjecture 2 from [22] which was mentioned above.

We deal with complexity questions related to feasible sets of mixed hypergraphs in Section 4. We prove that for any fixed finite sets of positive integers $A_{1} \subset A_{2}$, it is NP-hard to decide whether the feasible set of a given mixed hypergraph $H$ is equal to $A_{2}$ even if it is promised that $\mathcal{F}(H)$ is either $A_{1}$ or $A_{2}$. This theorem has several interesting corollaries: It is NP-complete to decide whether a given mixed hypergraph is colorable, it is both NP-hard and coNP-hard for a fixed non-empty finite set of positive integers $A$ to decide whether the feasible set of a mixed hypergraph is equal to $A$, it is both NP-hard and coNP-hard to decide whether the feasible set of a given mixed hypergraph is gap-free. This particular result was previously obtained in [11]. It was also known before that it is NP-hard to decide whether a given mixed hypergraph is uniquely colorable [20] and that it is NP-hard to compute the upper chromatic number even when restricted to several special classes of mixed hypergraphs $[1,10,12,19]$. 
There is also no polynomial-time $o(n)$-approximation algorithm for the lower or the upper chromatic number unless $P=N P$ where $n$ is the number of vertices of an input mixed hypergraph. We remark there is an $O\left(n \frac{(\log \log n)^{2}}{\log ^{3} n}\right)$-approximation algorithm for the chromatic number of ordinary graphs [5]. Recall that an algorithm for a maximization (minimization) problem is said to be $K$-approximation algorithm if it always finds a solution whose value is at least $\mathrm{OPT} / K$ (at most $K \cdot \mathrm{OPT}$ ) where OPT is the value of the optimum solution.

\section{Small realizations of feasible sets}

If $\mathcal{F}=\{m\}$, then the complete graph of order $m$ is the one-realization of $\mathcal{F}$ with the fewest number of vertices. In this section, we present a one-realization with few vertices for the case $|\mathcal{F}| \geq 2$ :

Theorem 2 Let $\mathcal{F}$ be a finite non-empty set of positive integers with $1 \notin \mathcal{F}$. There exists a mixed hypergraph with at most $|\mathcal{F}|+2 \max \mathcal{F}-\min \mathcal{F}$ vertices whose feasible set is $\mathcal{F}$ and every entry of its spectrum is 0 or 1 . The number of the edges of this mixed hypergraph is cubic in the number of its vertices.

Proof: The proof proceeds by induction on $\max \mathcal{F}$. If $2 \notin \mathcal{F}$, then let $H^{\prime}$ be a onerealization of $\mathcal{F}^{\prime}=\{i-1 \mid i \in \mathcal{F}\}$. Let $H$ be the mixed hypergraph obtained from $H^{\prime}$ by adding a vertex $x$ and $\mathcal{D}$-edges $\{x, v\}$ for all $v \in V\left(H^{\prime}\right)$. This operation was also used in [6] under the name "elementary shift". It is clear that proper $\ell$-colorings of $H$ are in one-to-one correspondence with proper $(\ell+1)$-colorings of $H^{\prime}$, since the color of the vertex $x$ has to be different from the color of any other vertex and it does not affect coloring of any edge except for the added $\mathcal{D}$-edges of size two. Hence, $H$ is one-realization of $\mathcal{F}$. The number of vertices of $H$ is at most $1+\left|\mathcal{F}^{\prime}\right|+2 \max \mathcal{F}^{\prime}-\min \mathcal{F}^{\prime} \leq|\mathcal{F}|+2 \max \mathcal{F}-\min \mathcal{F}$.

It remains to consider the case when $\min \mathcal{F}=2$. The case of $\mathcal{F}=\{2\}$ is described before Theorem 2. In the rest, we assume that $\max \mathcal{F}>2$. For this purpose, we define a mixed hypergraph $H$ with the vertex set $\left\{v_{2}^{+}, \ldots, v_{m}^{+}, v_{1}^{-}, \ldots, v_{m}^{-}, v_{1}^{\oplus}\right\} \cup\left\{v_{i}^{\oplus} \mid i \in \mathcal{F} \backslash\{2, m\}\right\}$ where $m=\max \mathcal{F}$. Let $\mathcal{F}(H)=\left\{c_{1}, \ldots, c_{k}\right\}, c_{1}<\ldots<c_{k}$, and set $c_{1}^{\prime}=1$ and $c_{i}^{\prime}=c_{i}$ for $2 \leq i \leq k$. Next, we describe the edges of $H$. The mixed hypergraph $H$ contains the following edges for each $l, 2 \leq l \leq k$ :

$$
\begin{aligned}
& \left\{v_{i}^{-}, v_{j}^{+}\right\} \text {is a } \mathcal{D} \text {-edge for } c_{l-1}^{\prime} \leq i \leq c_{l}^{\prime} \text { and } c_{l-1}^{\prime}<j \leq c_{l}^{\prime} \text { with } i \neq j \\
& \left\{v_{i}^{-}, v_{c_{l-1}^{\prime}}^{\oplus}\right\} \text { is a } \mathcal{D} \text {-edge for } c_{l-1}^{\prime}<i \leq c_{l}^{\prime} \\
& \left\{v_{i}^{+}, v_{i}^{-}, v_{j}^{+}\right\} \text {is a } \mathcal{C} \text {-edge for } c_{l-1}^{\prime}<i, j \leq c_{l}^{\prime} \text { and } i \neq j \\
& \left\{v_{i}^{+}, v_{i}^{-}, v_{c_{l-1}^{\prime}}^{\oplus}\right\} \text { is a } \mathcal{C} \text {-edge for } c_{l-1}^{\prime}<i \leq c_{l}^{\prime} \\
& \left\{v_{i}^{+}, v_{i}^{-}, v_{j}^{-}\right\} \text {is a } \mathcal{C} \text {-edge for } c_{l-1}^{\prime}<i, j \leq c_{l}^{\prime} \text { and } i \neq j \\
& \left\{v_{c_{l-1}^{\prime}}^{\oplus}, v_{c_{l-1}^{\prime}}^{-}, v_{j}^{+}\right\} \text {is a } \mathcal{C} \text {-edge for } c_{l-1}^{\prime}<j \leq c_{l}^{\prime} \\
& \left\{v_{c_{l-1}^{\prime}}^{\oplus}, v_{c_{l-1}^{\prime}}^{-}, v_{j}^{-}\right\} \text {is a } \mathcal{C} \text {-edge for } c_{l-1}^{\prime}<j \leq c_{l}^{\prime}
\end{aligned}
$$




$$
\begin{aligned}
& \left\{v_{c_{l}^{\prime}}^{+}, v_{c_{l}^{\prime}}^{-}, v_{c_{l}^{\prime}}^{\oplus}\right\} \text { is a } \mathcal{C} \text {-edge if } l<k \\
& \left\{v_{c_{l-1}^{\prime}}^{\oplus}, v_{c_{l}^{\prime}}^{+}, v_{c_{l}^{\prime}}^{\oplus}\right\} \text { is a } \mathcal{C} \text {-edge if } l<k \\
& \left\{v_{c_{l-1}^{\prime}}^{-}, v_{c_{l}^{\prime}}^{-}, v_{c_{l}^{\prime}}^{\oplus}\right\} \text { is a } \mathcal{D} \text {-edge if } l<k \\
& \left\{v_{i}^{-}, v_{j}^{+}, v_{j}^{-}\right\} \text {is a } \mathcal{D} \text {-edge for } 1 \leq i \leq c_{l}^{\prime}, c_{l-1}^{\prime}<j \leq c_{l}^{\prime} \text { and } i \neq j
\end{aligned}
$$

We prove that the mixed hypergraph $H$ has the properties claimed in the statement of the theorem. Note that $H$ is exactly the mixed hypergraph $H^{k}$ defined in the next paragraph. In the rest, we slightly abuse the notation and we call the $\mathcal{D}$-edges described in (1) just $\mathcal{D}$-edges (1) and we call other kinds of edges in a similar way.

Let $H^{l}$ be the mixed hypergraph obtained from by $H$ (for $l \geq 2$ ) restricting to the vertices $\left\{v_{2}^{+}, \ldots, v_{c_{l}^{\prime}}^{+}, v_{1}^{-}, \ldots, v_{c_{l}^{\prime}}^{-}, v_{1}^{\oplus}\right\} \cup\left\{v_{i}^{\oplus} \mid i \in \mathcal{F} \wedge 3 \leq i<c_{l}^{\prime}\right\}$. The edges of $H^{l}$ are those edges of $H$ which are fully contained in the vertex set of $H^{l}$. We prove that the following statements hold for all $l, 2 \leq l \leq k$ :

1. $\mathcal{F}\left(H^{l}\right)=\left\{c_{1}, \ldots, c_{l}\right\}$

2. Any proper coloring $c$ of $H^{l}$ with $c\left(v_{c_{l}^{\prime}}^{+}\right) \neq c\left(v_{c_{l}^{\prime}}^{-}\right)$uses less than $c_{l}$ colors. In addition, $c$ satisfies that $c\left(v_{c_{l-1}^{\prime}}^{\oplus}\right)=c\left(v_{c_{l}^{\prime}}^{+}\right), c\left(v_{c_{l-1}^{\prime}}^{-}\right)=c\left(v_{c_{l}^{\prime}}^{-}\right) \neq c\left(v_{c_{l}^{\prime}}^{+}\right)$.

3. Any proper coloring $c$ of $H^{l}$ with $c\left(v_{c_{l}^{\prime}}^{+}\right)=c\left(v_{c_{l}^{\prime}}^{-}\right)$uses exactly $c_{l}$ colors. In addition, the coloring $c$ colors the vertices $v_{1}^{-}, \ldots, v_{c_{l}}^{-}$with mutually different colors and the colors of $v_{i}^{-}$and $v_{i}^{+}$(and $v_{i}^{\oplus}$ if it exists) are the same.

4. There is exactly one proper coloring using exactly $\lambda$ colors for each $\lambda \in \mathcal{F}\left(H^{l}\right)$.

These four claims are proved simultaneously by induction on $l$.

We first deal with the case that $l=2$. Let $c$ be a proper coloring of $H^{2}$. If $c\left(v_{1}^{\oplus}\right) \neq$ $c\left(v_{1}^{-}\right)$, then this coloring uses exactly two colors on the vertices $v_{1}^{-}, \ldots, v_{c_{2}^{\prime}}^{-}, v_{1}^{\oplus}, v_{2}^{+}, \ldots, v_{c_{2}^{\prime}}^{+}$ due to the presence of $\mathcal{C}$-edges (6) and (7). Furthermore, the $\mathcal{D}$-edges (1) and (2) force that $c\left(v_{1}^{-}\right)=c\left(v_{2}^{-}\right)=\ldots=c\left(v_{c_{2}^{\prime}}^{-}\right)$and $c\left(v_{1}^{+}\right)=c\left(v_{2}^{+}\right)=\ldots=c\left(v_{c_{2}^{\prime}}^{+}\right)=c\left(v_{1}^{\oplus}\right)$ Thus, the vertices are colored as described in the second claim.

Let us now suppose that $c\left(v_{1}^{\oplus}\right)=c\left(v_{1}^{-}\right)$. If $c\left(v_{i}^{+}\right) \neq c\left(v_{i}^{-}\right)$for some $2 \leq i \leq c_{2}^{\prime}$, then $c\left(v_{1}^{\oplus}\right) \neq c\left(v_{1}^{-}\right)$due to the presence of $\mathcal{C}$-edges (4) and (5) and $\mathcal{D}$-edges (1) and (2). Thus $c\left(v_{i}^{+}\right)=c\left(v_{i}^{-}\right)$for all $2 \leq i \leq c_{2}^{\prime}$. The colors of $c\left(v_{i}^{-}\right)$for $1 \leq i \leq c_{2}^{\prime}$ are mutually distinct due to the presence of $\mathcal{D}$-edges (1) and (2). We may infer that any such coloring $c$ assigns $c_{2}^{\prime}$ colors to the vertices $v_{1}^{-}, \ldots, v_{c_{2}^{\prime}}^{-}, v_{1}^{\oplus}, v_{2}^{+}, \ldots, v_{c_{2}^{\prime}}^{+}$. Hence, the coloring $c$ uses exactly $c_{2}=c_{2}^{\prime}$ colors. This finishes the proof of all the four claims for $H^{2}$. It is straightforward to check that the two given colorings of $H^{2}$ are proper.

Let us prove the claims $1,2,3$ and 4 for $H^{l}(l \geq 3)$ assuming them proved for $H^{l-1}$. Consider a proper coloring $c$ of a mixed hypergraph $H^{l}$. If $c\left(v_{c_{l-1}^{\prime}}^{-}\right) \neq c\left(v_{c_{l-1}^{\prime}}^{+}\right)$, then the $\mathcal{C}$-edge $(8)$ and the $\mathcal{D}$-edge $(10)$ together with the second claim assure that $c\left(v_{c_{l-1}^{\prime}}^{+}\right)=c\left(v_{c_{l-1}^{\prime}}^{\oplus}\right)$. Note that in this case $c$ uses less than $c_{l-1}$ colors to color vertices of 
$H^{l-1}$. If $c\left(v_{c_{l-1}^{\prime}}^{-}\right)=c\left(v_{c_{l-1}^{\prime}}^{+}\right)$, then $c\left(v_{c_{l-2}^{\prime}}^{-}\right)=c\left(v_{c_{l-2}^{\prime}}^{\oplus}\right) \neq c\left(v_{c_{l-1}^{\prime}}^{-}\right)$. Thus, the color $c\left(v_{c_{l-1}^{\prime}}^{\oplus}\right)$ is either $c\left(v_{c_{l-1}^{\prime}}^{-}\right)=c\left(v_{c_{l-1}^{\prime}}^{+}\right)$or $c\left(v_{c_{l-2}^{\prime}}^{-}\right)=c\left(v_{c_{l-2}^{\prime}}^{\oplus}\right)$ due to the presence of the $\mathcal{C}$-edge $(9)$ (and both is possible). In both cases, the coloring $c$ must use exactly $c_{l-1}$ colors to color vertices of $H^{l-1}$.

We distinguish two cases (similar to those above): $c\left(v_{c_{l-1}^{\prime}}^{-}\right) \neq c\left(v_{c_{l-1}^{\prime}}^{\oplus}\right)$ and $c\left(v_{c_{l-1}^{\prime}}^{-}\right)=$ $c\left(v_{c_{l-1}^{\prime}}^{\oplus}\right)$. If $c\left(v_{c_{l-1}^{\prime}}^{-}\right) \neq c\left(v_{c_{l-1}^{\prime}}^{\oplus}\right)$, then the same argumentation as used before yields that $c\left(v_{c_{l-1}^{\prime}}^{-}\right)=\ldots=c\left(v_{c_{l}^{\prime}}^{-}\right)$and $c\left(v_{c_{l-1}^{\prime}}^{\oplus}\right)=c\left(v_{c_{l-1}^{\prime}+1}^{+}\right)=\ldots=c\left(v_{c_{l}^{\prime}}^{+}\right)$. On the other hand, if $c\left(v_{c_{l-1}^{\prime}}^{-}\right)=c\left(v_{c_{l-1}^{\prime}}^{\oplus}\right)$, then we can again infer that $c\left(v_{c_{l-1}^{\prime}}^{\oplus}\right)=c\left(v_{c_{l-1}^{\prime}}^{-}\right), c\left(v_{c_{l-1}^{\prime}+1}^{+}\right)=c\left(v_{c_{l-1}^{\prime}+1}^{-}\right) \neq$ $\ldots \neq c\left(v_{c_{l}^{\prime}}^{+}\right)=c\left(v_{c_{l}^{\prime}}^{-}\right)$. The colors $c\left(v_{c_{l-1}^{\prime}}^{-}\right), \ldots, c\left(v_{c_{l}^{\prime}}^{-}\right)$are also mutually distinct because of the $\mathcal{D}$-edges $(1)$ and $(2)$ and they are different from colors $c\left(v_{1}^{-}\right), \ldots, c\left(v_{c_{l-1}^{\prime}-1}^{-}\right)$due to the presence of $\mathcal{D}$-edges (11) and the third claim used for $H^{l-1}$. This proves the first, the second and the third claim for $H^{l}$. We again leave a straightforward check that all the described colorings are proper. As to the fourth claim: If $c\left(v_{c_{l-1}^{\prime}}^{-}\right)=c\left(v_{c_{l-1}^{\prime}}^{\oplus}\right)$, then exactly $c_{l-1}$ colors are used to color the vertices of $H^{l-1}$ and new $c_{l}-c_{l-1}$ colors are used to color the vertices of $v_{c_{l-1}^{\prime}+1}^{+}, \ldots, v_{c_{l}^{\prime}}^{+}$and $v_{c_{l-1}^{\prime}+1}^{-}, \ldots, v_{c_{l}^{\prime}}^{-}$due to the presence of $\mathcal{D}$-edges 11 . On the other hand, if $c\left(v_{c_{l-1}^{\prime}}^{-}\right) \neq c\left(v_{c_{l-1}^{\prime}}^{\oplus}\right)$, there exists unique extension of any proper coloring of $H^{l-1}$ to $H^{l}$. This finishes the proof of all the four claims on $H^{l}$.

We conclude that $H=H^{k}$ has the desired properties. The bound on the number of edges follows from the fact that each edge has size at most three.

We immediately have the following corollary of Theorems 1 and 2:

Corollary 1 There exists a polynomial-time algorithm which for a given set $\mathcal{F}$ decides whether it is a feasible set of some mixed hypergraph and if so it outputs a mixed hypergraph $H$ such that $\mathcal{F}(H)=\mathcal{F}$.

Proof: If $1 \notin \mathcal{F}$, then the algorithm returns the construction from Theorem 2. If $1 \in \mathcal{F}$ and $\mathcal{F}$ is not interval, then the algorithm returns that no such mixed hypergraph exists (Theorem 1). If $1 \in \mathcal{F}$ and $\mathcal{F}$ is an interval, then the algorithm outputs a mixed hypergraph consisting of $\max \mathcal{F}$ vertices and no edges.

\section{Realizations of spectra}

We first slightly alter the construction from Theorem 2:

Lemma 1 Let $\mathcal{F}=\left\{c_{1}, \ldots, c_{k}\right\}$ be a set of positive integers with $1 \notin \mathcal{F}$. There exists a mixed hypergraph $H^{*}$ with at most $2(|\mathcal{F}|+\max \mathcal{F})$ vertices which is a one-realization of $\mathcal{F}$. Moreover, $H^{*}$ contains $3 l$ vertices $w_{i}^{+}, w_{i}^{\oplus}, w_{i}^{\ominus}(1 \leq i \leq k)$ with the following property: Let $c$ be any proper coloring of $H^{*}$, then 
- The vertices $w_{i}^{+}, w_{i}^{\oplus}, w_{i}^{\ominus}$ are colored by c with exactly two colors for each $i$.

- $c\left(w_{i}^{\ominus}\right)=c\left(w_{i}^{+}\right) \neq c\left(w_{i}^{\oplus}\right)$ iff $c$ uses exactly $c_{i}$ colors.

- $c\left(w_{i}^{\ominus}\right) \neq c\left(w_{i}^{+}\right)=c\left(w_{i}^{\oplus}\right)$ iff $c$ does not use exactly $c_{i}$ colors.

Proof: If $\mathcal{F}=\{2\}$, then consider the following mixed hypergraph $H^{*}: V\left(H^{*}\right)=$ $\left\{w_{1}^{+}, w_{1}^{\oplus}, w_{1}^{\ominus}\right\}$ where $\left\{w_{1}^{\ominus}, w_{1}^{+}\right\}$is the only $\mathcal{C}$-edge of $H^{*}$ and $\left\{w_{1}^{\ominus}, w_{1}^{\oplus}\right\}$ is the only $\mathcal{D}$ edge of $H^{*}$.

Assume now that $2 \in \mathcal{F}$ and $\mathcal{F} \neq\{2\}$. The case $2 \notin \mathcal{F}$ is considered later. We extend the construction from the proof of Theorem 2. Let $H^{k}$ be the mixed hypergraph obtained in the construction and let us continue using notation from the proof of Theorem 2 . We add a vertex $v_{1}^{+}$together with a $\mathcal{C}$-edge $\left\{v_{1}^{+}, v_{1}^{-}\right\}$and a vertex $v_{c_{k}^{\prime}}^{\oplus}$ together with a $\mathcal{C}$-edge $\left\{v_{c_{k-1}^{\prime}}^{\oplus}, v_{c_{k}^{\prime}}^{\oplus}\right\}$. It is routine to check that the following two claims hold:

- $c\left(v_{c_{i}^{\prime}}^{-}\right)=c\left(v_{c_{i}^{\prime}}^{+}\right) \neq c\left(v_{c_{i}^{\prime}}^{\oplus}\right)$ iff $c$ uses exactly $c_{i}$ colors.

- $c\left(v_{c_{i}^{\prime}}^{+}\right)=c\left(v_{c_{i}^{\prime}}^{\oplus}\right)$ iff $c$ does not use exactly $c_{i}$ colors.

Let $w_{i}^{+}=v_{c_{i}^{\prime}}^{+}, w_{i}^{-}=v_{c_{i}^{\prime}}^{-}$and $w_{i}^{\oplus}=v_{c_{i}^{\prime}}^{\oplus}$. We add new vertices $w_{i}^{\ominus}$ for all $1 \leq i \leq k$ to the mixed hypergraph together with $\mathcal{C}$-edges $\left\{w_{i}^{\ominus}, w_{i}^{+}, w_{i}^{\oplus}\right\}$ and $\left\{w_{i}^{\ominus}, w_{i}^{+}, w_{i}^{-}\right\}$for all $1 \leq i \leq k, \mathcal{C}$-edges $\left\{w_{i}^{\ominus}, w_{i}^{-}, v_{1}^{-}\right\}$for all $2 \leq i \leq k$ and $\mathcal{D}$-edges $\left\{w_{i}^{\ominus}, w_{i}^{\oplus}\right\}$ for all $1 \leq i \leq k$. We further add a $\mathcal{C}$-edge $\left\{w_{1}^{\ominus}, w_{1}^{-}, v_{2}^{-}\right\}$. The resulting mixed hypergraph is $H^{*}$.

Let $c$ be a proper coloring of $H^{*}$. If $c\left(w_{i}^{+}\right) \neq c\left(w_{i}^{\oplus}\right)$ (and thus $c\left(w_{i}^{+}\right)=c\left(w_{i}^{-}\right)$), then the $\mathcal{C}$-edge $\left\{w_{i}^{\ominus}, w_{i}^{+}, w_{i}^{\oplus}\right\}$ and the $\mathcal{D}$-edge $\left\{w_{i}^{\ominus}, w_{i}^{\oplus}\right\}$ force the vertex $w_{i}^{\ominus}$ to have the color $c\left(w_{i}^{+}\right)=c\left(w_{i}^{-}\right)$(and this extension is possible) - this describes the case when the coloring $c$ uses exactly $c_{i}$ colors. Let us assume further $c\left(w_{i}^{+}\right)=c\left(w_{i}^{\oplus}\right)$. If $c\left(w_{i}^{+}\right) \neq c\left(w_{i}^{-}\right)$, then the $\mathcal{C}$-edge $\left\{w_{i}^{\ominus}, w_{i}^{+}, w_{i}^{-}\right\}$and the $\mathcal{D}$-edge $\left\{w_{i}^{\ominus}, w_{i}^{\oplus}\right\}$ force the vertex $w_{i}^{\ominus}$ to have the color $c\left(w_{i}^{-}\right)$ (and this extension is possible). If $c\left(w_{i}^{+}\right)=c\left(w_{i}^{\oplus}\right)=c\left(w_{i}^{-}\right)$, then the $\mathcal{C}$-edge $\left\{w_{i}^{\ominus}, w_{i}^{-}, v_{1}^{-}\right\}$ (the $\mathcal{C}$-edge $\left\{w_{1}^{\ominus}, w_{1}^{-}, v_{2}^{-}\right\}$in case $i=1$ ) and the $\mathcal{D}$-edge $\left\{w_{i}^{\ominus}, w_{i}^{\oplus}\right\}$ force the vertex $w_{i}^{\ominus}$ to have the color $c\left(v_{1}^{-}\right)$(the color $\left.c\left(v_{2}^{-}\right)\right)$. This requires that $c\left(v_{1}^{-}\right) \neq c\left(w_{i}^{+}\right)=c\left(w_{i}^{\oplus}\right)=c\left(w_{i}^{-}\right)$ $\left(c\left(v_{2}^{-}\right) \neq c\left(w_{i}^{\oplus}\right)\right.$ where $w_{i}^{\oplus}=v_{1}^{\oplus}$, since $i$ is 1 in this case). The last non-equality is assured by the presence of the $\mathcal{D}$-edge $(11)(\mathcal{D}$-edge $(2))$ in the construction of Theorem 2 . This implies that each coloring $c$ of $H^{k}$ can be uniquely extended to $H^{*}$.

It is straightforward to check that all the three properties stated by the lemma hold. The second and the third one are established due to the presence of a $\mathcal{C}$-edge $\left\{w_{i}^{\ominus}, w_{i}^{+}, w_{i}^{-}\right\}$ and a $\mathcal{D}$-edge $\left\{w_{i}^{\ominus}, w_{i}^{\oplus}\right\}(1 \leq i \leq k)$ and due to the analogous claims stated in the previous paragraph for $v_{c_{i}^{\prime}}^{+}$and $v_{c_{i}^{\prime}}^{\oplus}$. The first one is established by the presence of the $\mathcal{C}$-edges $\left\{w_{i}^{\ominus}, w_{i}^{+}, w_{i}^{-}\right\}$and $\mathcal{D}$-edges $\left\{w_{i}^{\ominus}, w_{i}^{\oplus}\right\}$.

The final case to consider is that $2 \notin \mathcal{F}$. In this case, we first construct a mixed hypergraph for $\mathcal{F}^{\prime}=\{i-1 \mid i \in \mathcal{F}\}$ and then add a new vertex $x$ together with $\mathcal{D}$-edges $\{x, v\}$ for all vertices $v$ as in the beginning of the proof of Theorem 2 . 
Lemma 2 Let $l$ be a given positive integer. There is a mixed hypergraph $H_{l}$ with three special vertices $w^{+}, w^{\ominus}, w^{\oplus}$ which satisfies: Let $c$ be any precoloring of $w^{+}, w^{\ominus}$ and $w^{\oplus}$ using two colors such that $c\left(w^{\ominus}\right) \neq c\left(w^{\oplus}\right)$, then:

- Any extension of $c$ to a proper coloring of $H_{l}$ uses no additional colors.

- If $c\left(w^{\ominus}\right) \neq c\left(w^{+}\right)=c\left(w^{\oplus}\right)$, then $c$ can be uniquely extended to a proper coloring of $H_{l}$.

- If $c\left(w^{\ominus}\right)=c\left(w^{+}\right) \neq c\left(w^{\oplus}\right)$, then $c$ can be extended to exactly $l$ different proper colorings of $H_{l}$.

The number of vertices of $H_{l}$ does not exceed $3+2\left\lfloor\log _{2} l\right\rfloor$.

Proof: The proof proceeds by induction on $l$. The statement is trivial for $l=1$. We distinguish two cases:

\section{- The number $l$ is even.}

Let $H_{l}$ be the mixed hypergraph obtained from $H_{l / 2}$ by adding a new vertex $x$, a $\mathcal{C}$-edge $\left\{w^{\oplus}, w^{\ominus}, x\right\}$ and a $\mathcal{D}$-edge $\left\{w^{\oplus}, w^{+}, x\right\}$. If $c\left(w^{\ominus}\right) \neq c\left(w^{+}\right)=c\left(w^{\oplus}\right)$, then $c$ can be extended uniquely to $H_{l / 2}$ and also to $x$, since the added edges force that $c(x)=c\left(w^{\ominus}\right)$. If $c\left(w^{\ominus}\right)=c\left(w^{+}\right) \neq c\left(w^{\oplus}\right)$, then $c$ can be extended to $l / 2$ different proper colorings to $H_{l / 2}$ and it can be extended by setting $c(x)$ to either $c\left(w^{\oplus}\right)$ or $c\left(w^{\ominus}\right)$. Altogether, we obtain $l$ different extensions.

\section{- The number $l$ is odd.}

Let $l=2 t+1$ and consider the mixed hypergraph $H_{t}$ with the properties described in the statement of the lemma. Let $w^{\prime+}, w^{\prime \oplus}, w^{\prime \ominus}$ be the special vertices of $H_{t}$. The mixed hypergraph $H_{l}$ is constructed as follows: We set the vertex $w^{\oplus}$ to be $w^{\prime \ominus}$ and $w^{\ominus}$ to $w^{\prime \oplus}$. In addition, new vertices $w^{+}$and $x$ are introduced. Now add $\mathcal{C}$-edges $\left\{w^{\oplus}, w^{\ominus}, w^{\prime+}\right\}$ and $\left\{w^{\oplus}, w^{\ominus}, x\right\}$, and $\mathcal{D}$-edges $\left\{w^{\oplus}, w^{+}, w^{\prime+}\right\}$ and $\left\{w^{\ominus}, w^{\prime+}, x\right\}$. If $c\left(w^{\ominus}\right) \neq c\left(w^{+}\right)=c\left(w^{\oplus}\right)$, then the added $\mathcal{C}$-edges and $\mathcal{D}$-edges force that $c\left(w^{++}\right)=$ $c\left(w^{\ominus}\right)$ and $c(x)=c\left(w^{\oplus}\right)$. The coloring $c$ can be uniquely extended to the remaining vertices of $H_{t}$. If $c\left(w^{\ominus}\right)=c\left(w^{+}\right) \neq c\left(w^{\oplus}\right)$, then $c\left(w^{\prime+}\right)$ can be either $c\left(w^{\oplus}\right)$ or $c\left(w^{\ominus}\right)$. We consider these two possibilities. If $c\left(w^{+}\right)$is $c\left(w^{\ominus}\right)$, then $c(x)$ has to be $c\left(w^{\oplus}\right)$ and $c$ can be uniquely extended to the remaining vertices of $H_{t}$. If $c\left(w^{\prime+}\right)$ is $c\left(w^{\oplus}\right)$, then $c(x)$ can be either $c\left(w^{\oplus}\right)$ or $c\left(w^{\ominus}\right)$ and $c$ can be extended in $t$ different ways to the remaining vertices of $H_{t}$. Thus, $c$ can be extended altogether in $2 t+1=l$ different ways.

The bound on the number of vertices of $H_{l}$ is obviously fulfilled in both the cases.

We combine Lemmas 1 and 2 to get the main result of this section: 
Theorem 3 Let $\left(r_{1}, \ldots, r_{m}\right)$ be any vector of non-negative integers such that $r_{1}=0$. Then there exists a mixed hypergraph with at most $2 m+2 \sum_{i=1, r_{i} \neq 0}^{m}\left(1+\left\lfloor\log _{2} r_{i}\right\rfloor\right)$ vertices such that its spectrum is equal to $\left(r_{1}, \ldots, r_{m}\right)$. Moreover, the number of edges of this mixed hypergraph is cubic in the number of its vertices.

Proof: Let $\mathcal{F}=\left\{j \mid r_{j} \neq 0\right\}$ and let $H^{*}$ be the mixed hypergraph from Lemma 1 . We keep the notation of Lemma 1 . We apply the following procedure for each $c_{i} \in \mathcal{F}\left(H^{*}\right)$ : We add a copy of $H_{r_{c_{i}}}$ from Lemma 2 to $H^{*}$ and we identify vertices $w_{i}^{+}$and $w^{+}, w_{i}^{\oplus}$ and $w^{\oplus}$ and $w_{i}^{\ominus}$ and $w^{\ominus}$. Lemmas 1 and 2 now yield that the spectrum of the just constructed mixed hypergraph is $\left(r_{1}, \ldots, r_{m}\right)$. The bound on the number of vertices easily follows from counting the number of the vertices of $H^{*}$ and the vertices of $H_{r_{c_{i}}}$ (and realizing that some of the vertices have been identified). The bound on the number of edges follows from the fact that each edge has size at most three.

\section{Complexity results}

The main theorem of this section is proved in a similar way as Theorem 3, except that instead of Lemma 2, we use the following lemma:

Lemma 3 Let $\Phi$ be a given formula with clauses of size three and let $n$ be the number of variables and $m$ the number of clauses of $\Phi$. There exists a mixed hypergraph $H_{\Phi}$ with three special vertices $w^{+}, w^{\ominus}, w^{\oplus}$ which satisfies: Let $c$ be any precoloring of $w^{+}, w^{\ominus}$ and $w^{\oplus}$ using two colors such that $c\left(w^{\ominus}\right) \neq c\left(w^{\oplus}\right)$, then:

- Any extension of c to a proper coloring of $H_{\Phi}$ uses no additional colors.

- If $c\left(w^{\ominus}\right) \neq c\left(w^{+}\right)=c\left(w^{\oplus}\right)$, then $c$ can always be extended to a proper coloring of $H_{\Phi}$.

- If $c\left(w^{\ominus}\right)=c\left(w^{+}\right) \neq c\left(w^{\oplus}\right)$, then $c$ can be extended to a proper colorings of $H_{\Phi}$ iff $\Phi$ is satisfiable.

$H_{\Phi}$ has $2 n+3$ vertices and $3 n+m$ edges.

Proof: Let $x_{1}, \ldots, x_{m}$ be the variables of the given formula. Let $H_{\Phi}$ be a mixed hypergraph with vertices $w^{+}, w^{\ominus}, w^{\oplus}, v_{1}^{\mathrm{T}}, v_{1}^{\mathrm{F}}, \ldots, v_{n}^{\mathrm{T}}, v_{n}^{\mathrm{F}}$ and the following edges:

- $\mathcal{C}$-edges $\left\{w^{\oplus}, w^{\ominus}, v_{i}^{\mathrm{T}}\right\}$ and $\left\{w^{\oplus}, w^{\ominus}, v_{i}^{\mathrm{F}}\right\}$ for $1 \leq i \leq n$,

- $\mathcal{D}$-edges $\left\{v_{i}^{\mathrm{T}}, v_{i}^{\mathrm{F}}\right\}$ for $1 \leq i \leq n$ and

- $\mathcal{D}$-edges $\left\{w^{\ominus}, w^{+}, w_{i}^{X}, w_{j}^{Y}, w_{k}^{Z}\right\}$ for each clause of the formula containing the variables $x_{i}, x_{j}$ and $x_{k}$ where $X=\mathrm{T}$ if the occurrence of $x_{i}$ in the clause is positive and $X=\mathrm{F}$ otherwise; $Y$ and $Z$ are set in the same manner. 
The bounds on the size of $H_{\Phi}$ are clearly fulfilled.

Any extension of a precoloring $c$ of the vertices $w^{+}, w^{\ominus}, w^{\oplus}$ with $c\left(w^{\ominus}\right) \neq c\left(w^{\oplus}\right)$ to the vertices $v_{1}^{\mathrm{T}}, v_{1}^{\mathrm{F}}, \ldots, v_{n}^{\mathrm{T}}, n_{n}^{\mathrm{F}}$ uses only the colors $c\left(w^{\ominus}\right)$ and $c\left(w^{\oplus}\right)$ due to the presence of $\mathcal{C}$-edges $\left\{w^{\oplus}, w^{\ominus}, v_{i}^{\mathrm{T}}\right\}$ and $\left\{w^{\oplus}, w^{\ominus}, v_{i}^{\mathrm{F}}\right\}$ for all $1 \leq i \leq n$. If $c\left(w^{\ominus}\right) \neq c\left(w^{+}\right)$, then all the $\mathcal{D}$-edges corresponding to the clauses of $\Phi$ are properly colored already by the precoloring and thus assigning all the vertices $v_{i}^{\mathrm{T}}$ the color $c\left(w^{\oplus}\right)$ and all the vertices $v_{i}^{\mathrm{F}}$ the color $c\left(w^{\ominus}\right)$ yields a proper extension of $c$.

Let us assume in the rest of the proof that $c\left(w^{\ominus}\right)=c\left(w^{+}\right)$. The color $c\left(w^{\oplus}\right)$ represents true and the color $c\left(w^{\ominus}\right)$ represents false in our construction. The presence of $\mathcal{D}$-edges $\left\{v_{i}^{\mathrm{T}}, v_{i}^{\mathrm{F}}\right\}$ assures that each variable and its negation have opposite values (recall that the value of $x_{i}$ is represented by the color of $\left.v_{i}^{\mathrm{T}}\right)$. The $\mathcal{D}$-edges $\left\{w^{\ominus}, w^{+}, v_{i}^{X}, v_{j}^{Y}, v_{k}^{Z}\right\}$ force that each clause contains at least one true literal (a vertex colored by the color $c\left(w^{\oplus}\right)$. Hence, $c$ can be extended to $H_{\Phi}$ iff there is a satisfying assignment of $\Phi$.

We now combine Lemmas 1 and 3 to get the following theorem:

Theorem 4 Let $A_{2}$ be a finite non-empty subset of $\{2,3, \ldots\}$ and $A_{1}$ a proper (possibly empty) subset of $A_{2}$. It is NP-hard to decide whether the feasible set of a given mixed hypergraph $\mathrm{H}$ is equal to $A_{2}$ even if it is promised that $\mathcal{F}(H)$ is either $A_{1}$ or $A_{2}$.

Proof: We present a reduction from the well-known NP-complete problem 3SAT. Let $\Phi$ be a given formula with $n$ variables and $H_{\Phi}$ the mixed hypergraph from Lemma 3 . Consider the mixed hypergraph $H^{*}$ from Lemma 1 for the set $\mathcal{F}=A_{2}=\left\{c_{1}, \ldots, c_{k}\right\}$. Let $A_{2} \backslash A_{1}=\left\{c_{i_{1}}, \ldots, c_{i_{k^{\prime}}}\right\}$. We create $\left|A_{2}\right|-\left|A_{1}\right|=k^{\prime} \geq 1$ copies of $H_{\Phi}$ and we identify the vertices $w^{\ominus}, w^{+}, w^{\oplus}$ of the $j$-th copy with the vertices $w_{c_{i_{j}}}^{\ominus}, w_{c_{i_{j}}}^{+}, w_{c_{i_{j}}}^{\oplus}$ of $H^{*}$. Let $H$ be the obtained mixed hypergraph.

It is easy to verify that $H$ can be constructed in time polynomial in the number of variables and clauses of the formula $\Phi$. In particular, the number of vertices of $H$ is at most $3 \cdot \max A_{2}+2 \cdot\left|A_{2} \backslash A_{1}\right| \cdot n$ and the number of its edges is cubic in the number of its vertices.

The mixed hypergraph $H$ has a strict $\ell$-coloring for $\ell \in A_{1}$ since any strict $\ell$-coloring of $H^{*}$ can be extended to the copies of $H_{\Phi}$ due to Lemma 3. Recall that $c\left(w_{c_{i_{j}}}^{\ominus}\right) \neq$ $c\left(w_{c_{i_{j}}}^{+}\right)=c\left(w_{c_{i_{j}}}^{\oplus}\right)$ for $1 \leq j \leq k^{\prime}$ for every strict $\ell$-coloring of $H^{*}$ with $\ell \in A_{1}$. On the other hand, $H$ has a strict $\ell$-coloring for $\ell \in A_{2} \backslash A_{1}$ iff $\Phi$ is satisfiable: Since it holds that $c\left(w_{c_{i_{j}}}^{\ominus}\right)=c\left(w_{c_{i_{j}}}^{+}\right) \neq c\left(w_{c_{i_{j}}}^{\oplus}\right)$ for every strict $c_{i_{j}}$-coloring $c$ of $H^{*}$, the coloring $c$ can be extended to the $j$-th copy of $H_{\Phi}$ iff $\Phi$ is satisfiable by Lemma 3 .

Several interesting computational complexity corollaries follow almost immediately. These results are new except for Corollary 4 which was proved in a weaker form in [11]:

Corollary 2 It is NP-complete to decide whether a given mixed hypergraph $H$ is colorable. 
Proof: This problem clearly belongs to the class NP. It is enough to set $A_{1}=\emptyset$ and $A_{2}=\{2\}$ in Theorem 4 to get the result.

Corollary 3 Let $A$ be a fixed finite subset of $\{2,3, \ldots\}$. It is coNP-hard to decide whether the feasible set of a given mixed hypergraph $H$ is equal to $A$. If $A \neq \emptyset$, then this problem is NP-hard, too.

Proof: The coNP-hardness is established by setting $A_{1}=A$ and $A_{2}$ to a proper finite superset of $A$ omitting 1 in Theorem 4. The NP-hardness is established by setting $A_{2}$ to $A$ and $A_{1}$ to a proper subset of $A$.

Corollary 4 It is both NP-hard and coNP-hard to decide whether the feasible set of a given mixed hypergraph $H$ is gap-free even for a mixed hypergraph $H$ with $\bar{\chi}(H)=4$.

Proof: The NP-hardness is established by setting $A_{1}=\{2,4\}$ and $A_{2}=\{2,3,4\}$ in Theorem 4. The coNP-hardness is established by setting $A_{1}=\{4\}$ and $A_{2}=\{2,4\}$.

Corollary 5 There is no polynomial-time o(n)-approximation algorithm for the lower or the upper chromatic number of a mixed hypergraph where $n$ is the number of its vertices unless $P=N P$.

Proof: Suppose that there is a polynomial-time $f(n)$-approximation algorithm for the lower chromatic number where $f(n) \in o(n)$ and $n$ is the number of vertices of a given mixed hypergraph. Let $\Phi$ be a given formula with clauses of size three with $N$ variables. Choose $m$ such that $m>2 \cdot f(3 m+2 N)$. It is not hard to see that there is such an integer $m \in O(N)$ since $f(n) \in o(n)$. Let $H$ be the mixed hypergraph from the construction of Theorem 4 for $A_{1}=\{m\}$ and $A_{2}=\{2, m\}$. Note that the number of vertices of $H$ is at most $3 k+2 N$. The approximation algorithm for the lower chromatic number outputs a number which is less then $m$ iff the feasible set of the input mixed hypergraph is $A_{2}$. Recall that $\mathcal{F}(H)=A_{2}$ iff $\Phi$ is satisfiable. Hence, the existence of the polynomial-time $o(n)$-approximation algorithm implies that $3 \mathrm{SAT} \in P$ and consequently $P=N P$. The non-existence (unless $\mathrm{P}=\mathrm{NP}$ ) of a polynomial-time $o(n)$-approximation algorithm for the upper chromatic number can be proved similarly. 


\section{Conclusion}

There exists a mixed hypergraph whose feasible set is $\mathcal{F}$ for any set $\mathcal{F}$ of positive integers with $1 \notin \mathcal{F}$. We proved that there exists a mixed hypergraph whose spectrum is $\left(r_{1}, \ldots, r_{m}\right)$ for any vector $\left(r_{1}, \ldots, r_{m}\right)$ of non-negative integers such that $r_{1}=0$. The number of vertices of the smallest mixed hypergraph which is a realization of a given set $\mathcal{F}$ has been substantially decreased from exponential to linear in $\max \mathcal{F}$. But the following question has not been answered: What is the number of vertices of the smallest mixed hypergraph whose feasible set is equal to a given set $\mathcal{F}$ ? Or even, what is the number of vertices of the smallest mixed hypergraph whose spectrum is equal to a given spectrum $\left(r_{1}, \ldots, r_{m}\right)$ ? The answer to any of these questions probably requires some very fine analysis.

We have not dealt with mixed hypergraphs containing only $\mathcal{C}$-edges in this paper. It is clear that if $r_{1} \neq 0$ (this is equivalent to the fact that a mixed hypergraphs contains only $\mathcal{C}$-edges $)$, then $r_{1}=1$. Furthermore, $r_{2}=\left(2^{n}-2\right) / 2$ for some $n$ since $\mathcal{C}$-edges of size two can be contracted without affecting the spectrum and any two-coloring of a mixed hypergraph on $n$-vertices with no $\mathcal{D}$-edges and with no $\mathcal{C}$-edges of size two is proper. This leads to the following problem: What are necessary and sufficient conditions for a vector $\left(r_{1}, \ldots, r_{m}\right)$ with $r_{1}=1$ to be the spectrum of a mixed hypergraph?

\section{Acknowledgement}

The author would like to thank Jiří Sgall for suggesting an easy way to prove Lemma 2, Jan Kratochvíl for helpful discussions, Zdeněk Dvořák for a careful reading of a preliminary version of this paper and the anonymous referee for helpful comments on the presentation of the results and pointing out several related references.

\section{References}

[1] C. Arbib, M. Flammini: On the upper chromatic number of $\left(v_{3}, b_{2}\right)$-configurations, Discrete Appl. Math. 120 (2002), 3-12.

[2] C. Colbourn, J. Dinitz, A. Rosa: Bicoloring Triple Systems, Electronic J. Combin. 6(1) (1999), \#R25.

[3] Ch. J. Colbourn, A. Rosa: Triple Systems, Clarendon Press, Oxford (1999), sect. 18.6. Strict colouring and the upper chromatic number, 340-341.

[4] Z. Dvořák, D. Král': On Planar Mixed Hypergraphs, Electronic J. Combin. 8 (1) (2001), \#R35.

[5] M. M. Halldórsson: A still better performance guarantee for approximate graph coloring, Inform. Process. Lett. 45 (1993), 19-23. 
[6] T. Jiang, D. Mubayi, Zs. Tuza, V. Voloshin, D. B. West: The chromatic spectrum of mixed hypergraphs, Graphs and Combin. 18(2) (2002), 309-318.

[7] D. Kobler, A. Kündgen: Gaps in the chromatic spectrum of face-constrained plane graphs, Electronic J. Combin. 8 (1) (2001), \#N3.

[8] D. Král': A counter-example to Voloshin's hypergraph co-perfectness conjecture, Australasian Journal on Combinatorics 27 (2003), 253-262.

[9] D. Král': Mixed hypergraphs and other graph coloring problems, submitted. Available as ITI report 2003-160.

[10] D. Král', J. Kratochvíl, A. Proskurowski, H.-J. Voss: Coloring mixed hypertrees, to appear in Discrete Appl. Math.

[11] D. Král', J. Kratochvíl, H.-J. Voss: Complexity Note on Mixed Hypergraphs, in: Proc. 26th International Symposium, Mathematical Foundations of Computer Science 2001 (MFCS'01), LNCS vol. 2136 (2001), Springer-Verlag, 474-486.

[12] D. Král', J. Kratochvíl, H.-J. Voss: Mixed Hypegraphs with Bounded Degree: EdgeColoring of Mixed Multigraphs, Theoretical Comp. Science 295 (2003), 263-278.

[13] D. Král', J. Kratochvíl, H.-J. Voss: Mixed hypercacti, to appear in Discrete Math..

[14] J. Kratochvíl, Z. Tuza, M. Voigt: New trends in the theory of graph colorings: Choosability and list coloring, in: Contemporary Trends in Discrete Mathematics (from DIMACS and DIMATIA to the future), DIMACS Series in Discrete Mathematics and Theoretical Computer Science, vol. 49, American Mathematical Society, Providence, RI (1999), 183-197.

[15] A. Kündgen, E. Mendelsohn, V. Voloshin: Colouring planar mixed hypergraphs, Electronic J. Combin. 7 (2000), \#R60.

[16] H. Lefmann, V. Rödl, R. Thomas: Monochromatic vs. multicolored paths, Graphs Combin. 8 (1992), 323-332.

[17] L. Milazzo, Zs. Tuza: Upper chromatic number of Steiner triple and quadruple systems, Discrete Math. 174 (1997), 247-259.

[18] L. Milazzo, Zs. Tuza: Strict colorings for classes of Steiner triple systems, Discrete Math. 182 (1998), 233-243.

[19] R. Scapellato, V. Voloshin: Some results on coloring of mixed hypergraphs, Rend. Semin. Mat. Messina Ser. II 205 (1999), 149-165.

[20] Zs. Tuza, V. Voloshin, H. Zhou: Uniquely colorable mixed hypergraphs, Discrete Math. 248 (2002), 221-236. 
[21] V. Voloshin: Coloring mixed hypergraphs: Theory, algorithms and applications, AMS, Providence, 2002.

[22] V. Voloshin: On the upper chromatic number of a mixed hypergraph, Australasian Journal of Combin. 11 (1995), 25-45.

[23] V. Voloshin: The mixed hypergraphs, Computer Science Journal of Moldova 1 (1993), 45-52. 